Results A total of eight patients were enrolled. Patients had advanced or recurrent leiomyosarcoma, carcinosarcoma, lowgrade endometrial stromal sarcoma (ESS), synovial sarcoma, and undifferentiated sarcoma. Urinary obstruction (87.5\%) was the most common presentation before the surgery. Complete resection (R0) was achieved five (62.5\%) patients. Median Operative time was 6 (range, 3-22) hours. Transfusion was performed in six patients (75\%) with median of 2.5 pack of RBC. Four patients needed postoperative intensive care for median of two days (range, 0-8) but there was no operationassociated mortality or severe life-threatening morbidity. Median pelvic control duration was 6 (range, 3-64) months, although disease progression was observed in other extrapelvic areas where preoperatively assessed to be broadly distributed and impossible to be completely resected. Interestingly, one patients with progression disease (PD) showed 16 months of pelvic control duration. One patient showed no recurrence after the surgery (10\%) and another patient showed stable disease (SD, 10\%). Median OS after LEER was 6 (6-65) months.

Conclusions LEER is feasible for surgical control of the pelvic sidewall tumor with acceptable complications.

\section{EPV243/\#63 SURGICAL AND ADJUVANT TREATMENTS FOR UTERINE PECOMA}

${ }^{1} \mathrm{G}$ Bogani*, ${ }^{2} \mathrm{~A}$ Gronchi, ${ }^{1} \mathrm{~A}$ Ditto, ${ }^{1} \mathrm{~F}$ Raspagliesi. ${ }^{1}$ Fondazione IRCCS Istituto Nazionale dei Tumori di Milano, Gynecologic Oncology, Milano, Italy; ${ }^{2}$ Fondazione IRCSS Istituto Tumori di Milano, Surgical Oncology, Milano, Italy

\subsection{6/ijgc-2021-IGCS.314}

Objectives Perivascular epitheliod cell tumors (PEComas) are rare mesenchymal neoplasms. Uterine PEComa is extremely rare and only limited evidence is still available.

Methods Charts of consecutive patients who had treatment (between 01/01/2010 and 12/31/2020) for newly diagnosed uterine PEComas were retrieved. Five-year outcomes were assessed using Kaplan-Meier and Cox hazard models.

Results Data of 23 patients with newly diagnosed PEComas were analyzed. Mean (SD) patients' age was 52 (14) years. Twenty-two patients had a surgical cytoreductive attempt. In one case surgery was not performed due to the presence of an extra-abdominal spread. Overall, seven (30\%) patients had disease outside the uterus at the time of surgery. Complete cytoreduction (no macroscopic residual tumor) was achieved in 19 patients. Eleven (48\%) patients had adjuvant treatments, consisting in anthracycline-based chemotherapy $(n=4)$,gemcitabine-based chemotherapy $(n=2)$, mTOR inhibitors $(n=4)$ and hormonal treatment $(n=1)$. Median (range) follow-up as 23 (2, 99) months. Eleven (48\%) recurrences occurred with a mean (SD) progression free-survival of 14 (11) months. After a median (range) follow-up of 23 (2-99) months, nine (39\%) patients died of disease. Residual tumor at surgery was the only factor correlating with the risk of developing recurrent disease $(p=0.023)$ and worse overall survival $(p=0.014)$. In our small series, stage of disease and adjuvant therapy administration had no impact on survival outcomes.

Conclusions Uterine PEComa represents a rare and aggressive entity. Molecular/genomic profiling of the disease is necessary to predict response to treatment. Further collaborative investigations are warranted to assess the role of various prognostic factors and evaluate the most effective surgical and medical treatment modalities

\section{EPV244/\#93 MALIGNANCIES IN TRANSPLANT PATIENTS: AN ATYPICAL PRESENTATION AND COURSE OF OVARIAN CARCINOMA - A CASE REPORT}

1) Verhaegen*, ${ }^{2} \mathrm{XB}$ Trinh, ${ }^{2} \mathrm{~B}$ Bracke, ${ }^{3} \mathrm{~V}$ Hartman, ${ }^{4} \mathrm{E}$ Philipse, ${ }^{5} \mathrm{G}$ BroeckX, ${ }^{6} \mathrm{~K}$ Storm,

${ }^{7}$ I Vermeiren. ${ }^{1}$ UZA, Gynaecology and Obstetrics, Edegem, Belgium; ${ }^{2}$ UZA, Gynaecological Oncology, Edegem, Belgium; ${ }^{3}$ UZA, Hepatobiliary Surgery, Edegem, Belgium; ${ }^{4}$ UZA, Nephrology, Edegem, Belgium; ${ }^{5}$ UZA, Pathology, Edegem, Belgium; ${ }^{6}$ UZA, Genetics, Edegem, Belgium; ' University of Antwerp, Gynaecological Oncology, Edegem, Belgium

\subsection{6/ijgc-2021-IGCS.315}

Objectives Donor-transmitted malignancies are rare due to the strict selection criteria for donors. Diagnosis is challenging because they often have an atypical presentation and a poor response to treatment.

Methods We present the case of a woman who was diagnosed with a donor-transmitted carcinoma after kidney transplantation.

Results Two years after kidney transplantation, a 61-year-old woman was diagnosed with a FIGO stage IIIB Mullerian ovarian cancer. Treatment with neo-adjuvant chemotherapy was started and complicated due to the use of immunosuppressants. An interval-debulking procedure showed poor response to chemotherapy and an optimal debulking could not be achieved. Pathology revealed a high grade tumor with immunohistochemistry suggestive for lung carcinoma. However, a PET-CT did not indicate any pulmonary disease. Due to the atypical presentation, immunohistochemistry results and untraceable primary tumor additional genetic DNA profiling was performed to further investigate the origin. A Y-chromosome specific marker revealed that the tumor originated from the donor-transplant. The oncological treatment and immunosuppressants were discontinued. The kidney transplant was surgically removed and hemodialysis was initiated. The body's own immune response led to a clinical, biochemical and radiological complete response and patient has no evidence of disease after 1 year of follow-up.

Conclusions This case report illustrates the diagnostic and therapeutic challenges of cancer in transplant-patients. We suggest that DNA profiling should be standard procedure in transplant patients presenting with metastatic disease. Although donor-transmitted malignancies are a very rare finding, awareness is critical since it can have life-saving clinical implications.

\section{EPV245/\#230 INCREASING INCIDENCE OF OVARIAN AND UTERINE CARCINOSARCOMA: A UNITED STATES CANCER STATISTICS STUDY}

1/ Tunnage ${ }^{*},{ }^{2} \mathrm{C}-1$ Liao, ${ }^{3} \mathrm{D}$ Lewis, ${ }^{4} \mathrm{MA}$ Caesar, ${ }^{5} \mathrm{~A}$ Chan, ${ }^{6} \mathrm{D}$ Lee, ${ }^{5} \mathrm{~A}$ Rohatgi, ${ }^{7} \mathrm{~K}$ Darcy, ${ }^{7} \mathrm{C}$ Tian, ${ }^{1} \mathrm{~L}$ Boyd, ${ }^{8} \mathrm{D}$ Kapp, ${ }^{9} \mathrm{~J}$ Chan. ${ }^{1}$ New York University, Gynecologic Oncology, New York, USA; ${ }^{2}$ Kaohsiung Veterans General Hospital, Obstetrics and Gynecology, Kaohsiung City, Taiwan; ${ }^{3}$ MedStar Washington Hospital Center, Obstetrics and Gynecology, Washington, USA; ${ }^{4}$ California Pacific Medical Center, Research Institute, San Francisco, USA; ${ }^{5}$ Palo Alto Medical Foundation Research Intitute, Obstetrics and Gynecology, Palo Alto, USA; ${ }^{6}$ Palo Alto Medical Foundation, Research Institute, Palo Alto, USA; ${ }^{7}$ Gynecologic Cancer Center of Excellence, Obstetrics and Gynecology, Washington, USA; ${ }^{8}$ Stanford University School of Medicine, Department of Radiation Oncology, Stanford, USA; ${ }^{9}$ California Pacific Medical Center, Obstetrics and Gynecology, San Francisco, USA

\subsection{6/ijgc-2021-IGCS.316}

Objectives To identify trends in the incidence of ovarian and uterine carcinosarcoma. 